\title{
Tecnura
}

INVESTIGACIÓN

\section{Análisis de expansión de una microrred en una zona no interconectada}

\section{Expansion analysis of a microgrid in a non-interconnected zone}

\author{
Daniel Nicolás González Cely ${ }^{\mathbb{D}}$, Alejandro Muñoz Rincón ${ }^{2}$ iD, Maximiliano Bueno López ${ }^{3}$ (D)
}

Cómo citar: González C., D.N., Muñoz R., A. y Bueno L., M. (2020). Análisis de expansión de una microrred en una zona no interconectada. Tecnura, 24(63) 40-56. DOI: https://doi.org/10.14483/22487638.15480

Fecha de de recepción: 10 de junio de 2019

\section{RESUMEN}

Contexto: En zonas no interconectadas, la utilización de microrredes se ha convertido en la única alternativa que tienen las personas para acceder al servicio de energía eléctrica. Debido a esto, es necesario optimizar estos sistemas y, de ser posible, expandir las ya existentes para aumentar la cobertura y de esta manera apoyar el cumplimiento del Objetivo de Desarrollo Sostenible 7, planteado por Naciones Unidas: energía asequible y no contaminante.

Metodología: Se presentan dos casos de expansión para una microrred basada en un sistema de generación fotovoltaica que se encuentra en una zona no interconectada de Colombia. Se realiza el análisis de generación eléctrica del sistema mediante Matlab/Simulink, mientras que el análisis de pequeña señal para verificar la estabilidad es ejecutado a través de Neplan. Así se logra establecer una metodología que permite definir la capacidad de expansión de una microrred.

Resultados: A partir del análisis de estabilidad de pequeña señal realizado en Neplan se obtienen los valores propios del sistema, tanto de la micro-
Fecha de de aceptación: 18 de octubre de 2019

rred actual como de los dos casos de expansión, con el fin de garantizar la estabilidad en la microrred ante tres escenarios diferentes: cargas no lineales, aumento de potencia demandada y generada.

Conclusiones: En los sistemas de generación solar fotovoltaica ubicados en zonas no interconectadas y operando en modo isla, los inversores influyen en la estabilidad de frecuencia considerablemente, debido a que la reducida inercia en estos sistemas conduce a una variación más rápida de este parámetro. Por esto, el análisis de estabilidad de pequeña señal es el método indicado para estudiar el comportamiento de microrredes ante variaciones de potencia demandada y generada, particularmente en los sistemas que operan en las zonas no interconectadas, porque ante pequeñas perturbaciones es posible identificar los cambios en la dinámica del sistema.

Palabras clave: análisis de pequeña señal; generación distribuida; microrredes; sistema solar fotovoltaico.

\section{ABSTRACT}

Context: The use of microgrids in non-interconnected areas has become the only alternative that people living in these regions have to access the

1 Ingeniero electricista. Universidad de La Salle. Bogotá, Colombia. Contacto: danielngonzalez28@unisalle.edu.co ORCID: https://orcid.org/0000-0003-2500-4641

2 Ingeniero electricista. Universidad de La Salle. Bogotá, Colombia. Contacto: amunoz44@unisalle.edu.co ORCID: https://orcid.org/0000-0003-1438-0721

Ingeniero electricista, magíster en Ingeniería Eléctrica, doctor en Ingeniería. Docente en el Departamento de Electrónica, Instrumentación y Control. Universidad del Cauca. Popayán, Colombia. Contacto: mbuenol@unicauca.edu.co

ORCID: https://orcid.org/0000-0002-7959-9962 
electricity service. Because of this it is necessary to optimize the use of these systems and if it is possible to expand existing ones to increase coverage and, in that way, support the implementation of the sustainable development goal 7 set by United Nations: Ensure access to affordable, reliable, sustainable and modern energy for all.

Methodology: In this paper, we present two cases of expansion for a microgrid based on a photovoltaic generation system that is located in a non-interconnected area of Colombia. The analysis of the electrical generation of the system is carried out using Matlab/Simulink and the small signal analysis to evaluate the stability is executed in Neplan. Based on the above, it is possible to establish a methodology that allows to define the expandability of a microgrid.

Results: From the small signal stability analysis performed in Neplan, the system's own values are obtained, both from the current microgrid and from the two cases of expansion, this in order to guarantee the property of stability in the micro grid in three different scenarios; non-linear loads, increased power demanded and generated.

Conclusions: In photovoltaic systems located in non-interconnected areas and operating in island mode the inverters influence frequency stability considerably, because the reduced inertia in these systems leads to a faster frequency variation. This is why the analysis of small signal stability is the method indicated to study the behavior of microgrids before variations in demand and generated power, particularly in systems that operate in areas not interconnected, because with small disturbances it is possible to identify the changes in the dynamics of the system.

Keywords: small signal stability analysis; microgrids; distributed generation; photovoltaic system.

\section{INTRODUCCIÓN}

Una microrred es un sistema de energía integrado que consta de cargas interconectadas y recursos de energía distribuida, que puede funcionar en paralelo a la red convencional o de forma aislada (Stevanoni, De Grève, Vallée y Deblecker, 2019). Las microrredes surgen como un sistema que beneficia la operación y cobertura de los sistemas de potencia para economías en desarrollo (Louie, 2018; Correa, Marulanda y Panesso, 2017). Es el caso del Resguardo Indígena Calle Santa Rosa, que aprovecha los beneficios de una microrred basada en un sistema de generación solar fotovoltaica para cubrir algunos servicios básicos. En Bueno-López y Garzon-Lemos (2017) se presenta en detalle la estructura de la microrred mencionada, la cual básicamente satisface algunas necesidades para refrigeración de alimentos y un centro educativo. También posee un sistema de monitoreo de las baterías que permite gestionar la demanda (Aabrek, Forseth, Bueno-López y Molinas,
2018). Sin embargo, la microrred no cuenta con la capacidad suficiente para abastecer la demanda de toda la comunidad; por esto se analiza la posibilidad de expandir el sistema. Uno de los desafíos a resolver es el de la estabilidad, ya que para los sistemas solares la naturaleza variable de la generación no tiene en muchos casos ningún impacto considerable en la estabilidad de frecuencia; pero, las características constructivas de los inversores fotovoltaicos influyen en la estabilidad de frecuencia considerablemente, es decir, la reducida inercia de estos sistemas conduce a un cambio de rango de frecuencia más rápido y a unas caídas de frecuencia más profundas (Song, Hill y Liu, 2019; Giral, Celedón, Galvis y Zona, 2017).

Para solucionar la problemática mencionada, se realiza una investigación de los campos de indagación relacionados con los sistemas fotovoltaicos y la estabilidad en microrredes. La revisión de Bastidas, Oviedo y Solano (2017) muestra los problemas de estabilidad que se encuentran habitualmente en una microrred, 
organizados según la naturaleza de sus causas: pequeña señal, transitoria y de tensión. Este mismo estudio plantea estrategias de dimensionado y posicionamiento de unidades de generación distribuida por medio de las cuales se mejora la estabilidad del sistema.

Majumder (2013) investiga algunos aspectos de la estabilidad en microrredes, identifica varias razones de los problemas de estabilidad en varias de estas y describe el enfoque generalizado para mejorar la estabilidad del sistema. Los aspectos transitorios, pequeña señal y estabilidad del voltaje en cada tipo de microrred los relaciona junto con el alcance de las mejoras.

Por su parte, Ramírez y Aquino (2009) presentaron una metodología y algunos resultados en el análisis de estabilidad de pequeña señal del Sistema Eléctrico de Pereira, trabajo que fue realizado por medio del software Neplan. También, utilizaron el método de análisis modal para el estudio de estabilidad de pequeña señal del sistema. A partir de este estudio fue posible concluir que dicho software ofrece varias ventajas frente a otros similares para estos análisis.

En cambio, He, Wu, Xu y Guerrero (2019) observaron la estabilidad de una microrred considerando variaciones en las unidades de generación, desde el enfoque de control automático.

En este artículo se propone una expansión para una microrred aislada, de tal forma que el sistema sea estable ante diferentes perturbaciones que puedan presentarse ante su operación normal; es decir, aumentar la cobertura del sistema conservando las condiciones técnicas necesarias de operación. Así, fue posible obtener diferentes escenarios y se analizaron los resultados; luego se determinaron las condiciones que debe tener la microrred para operar satisfactoria- mente sin perder la estabilidad y, adicionalmente, aumentar la confiabilidad.

\section{METODOLOGÍA}

Con el objetivo de brindar una guía para analizar la posible expansión de una microrred, a continuación se esboza la información que debe ser considerada en este proceso.

\section{Recolectar información de la zona no inter- conectada (ZNI)}

Se debe disponer información específica de la zona en la cual se encuentra ubicada la microrred, específicamente se busca información acerca de:

- $\quad$ Microrred instalada: identificar la ZNI y si se encuentra instalada alguna microrred de tipo solar fotovoltaico.

- Condiciones de la carga: número de usuarios, ubicación y distancia entre ellos; área promedio de vivienda; área disponible para instalación de generadores y almacenadores, y aplicación del servicio de energía.

- Condiciones de la zona: temperatura ambiente mínima, máxima y promedio; altura sobre el nivel del mar; densidad de descargas atmosféricas; resistividad aparente del terreno; identificación de ambientes salinos o húmedos, y usos del suelo.

\section{Identificar topología de la microrred}

Se debe determinar la topología de la microrred, es decir ubicación y distribución de los equipos de generación, configuración y trazado de la red de distribución, longitudes de ramales, niveles de voltaje, calibre de conductores y toda la información pertinente para elaborar un diagrama unifilar de la red, y asimismo modelarla y simularla. 


\section{Estimar nuevos perfiles de carga}

A partir del diagrama unifilar de la red y su respectiva modelación y simulación, se estiman nuevos usuarios que puedan conectarse a la red, con el fin de lograr mayor cobertura. Cabe resaltar que se deben mantener las condiciones técnicas de la red y no llevarlas a niveles de funcionamiento que no pueda cumplir.

\section{Determinar alternativas de expansión}

Con la modelación y simulación de la microrred original y posibles nuevos perfiles de carga, se determina qué alternativas de expansión se pueden llevar a cabo, ya sea aumentando el nivel de generación o, en caso de que existan, acoplando dos sistemas independientes en la zona, para aumentar la generación y lograr así mayor cobertura.

\section{Evaluación de los equipos de la microrred existente}

A partir de las alternativas de expansión planteadas es necesario e importante determinar la capacidad de los equipos y verificar si cumplen con las condiciones para la expansión, es decir: capacidad del banco de baterías, potencia del inversor, potencia del regulador y demás.

\section{Realizar análisis de la expansión}

Una vez escogidas las alternativas de expansión se realiza su respectivo análisis, para así modelar y simular el nuevo sistema diseñado, y determinar si su funcionamiento es correcto. Esto con ayuda del parámetro de estabilidad en microrredes y puntualizando en el análisis de pequeña señal que el software Neplan permite realizar.

\section{DESCRIPCIÓN DEL SISTEMA DE PRUEBAS}

La microrred empleada para el estudio de caso se describe en la tabla 1. A partir de esta información se modela y se realiza la simulación, de manera que se pueda analizar el sistema actual $y$, posteriormente, planear los posibles casos de expansión (Bueno-López y Garzón-Lemos, 2017).

\section{Simulación microrred actual}

\section{Sistema de energización en centro educativo}

La microrred del centro educativo tiene una estructura con un sistema fotovoltaico de 1500 W y una carga con un consumo de 750 W. El sistema fotovoltaico se implementó con un banco de baterías y un inversor, como se muestra en la figura 1. La simulación del arreglo fotovoltaico de la escuela y del centro de refrigeración se realizó en MatlabR2016a/Simulink.

El resultado representa que la demanda de energía de la escuela es cubierta por los paneles fotovoltaicos en los periodos donde la irradiación es mayor o igual a $1000 \mathrm{~W} / \mathrm{m}^{\wedge} 2$. La

Tabla 1. Descripción del sistema actual

\begin{tabular}{ccc} 
Sistema & Generación distribuida & Características de funcionamiento \\
Sistema de refrigeración & Energía solar PV & $\begin{array}{c}\text { El sistema alimenta una carga DC de 830 W. La poten- } \\
\text { cia máxima que puede generar es de 1000 W. }\end{array}$ \\
\hline Escuela & Energía solar PV & $\begin{array}{c}\text { El sistema alimenta una carga de 750 W a 120 V / 60 } \\
\text { Hz. La potencia máxima que puede ser generada es de } \\
1500 \text { W. }\end{array}$ \\
\hline
\end{tabular}

Fuente: elaboración propia. 
figura 2. muestra la potencia máxima obtenida a partir de los parámetros indicados en la tabla 2.

\section{Simulación en centro de refrigeración}

La microrred propuesta para el centro de refrigeración tiene un sistema fotovoltaico de 1000 W. La figura 3. muestra el diagrama unifilar para esta red la cual no contiene inversor, ya que todas las cargas son de tipo
DC. La carga se determina al observar la figura 4 , donde es posible ver la potencia que necesita un refrigerador bajo un patrón de uso residencial típico dadas las temperaturas variables. Con una temperatura promedio de $29{ }^{\circ} \mathrm{C}$ el consumo de energía por día es aproximadamente de $200 \mathrm{~W} \mathrm{~h}$ /día, lo que corresponde a un consumo de energía de 8,33 W h (Aabrek et al., 2018). a.

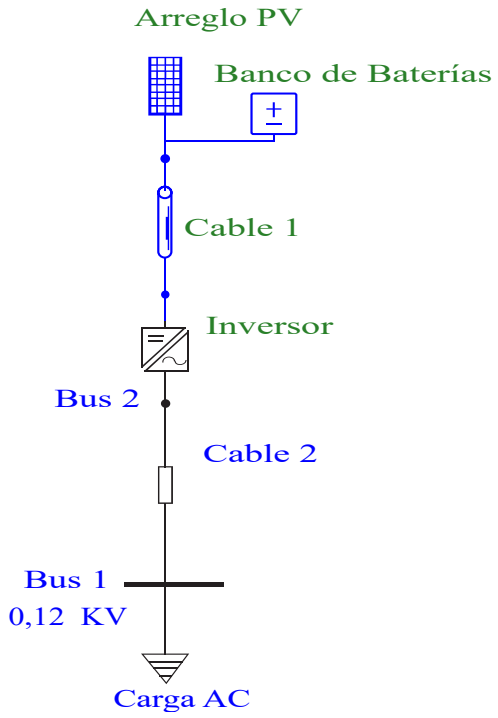

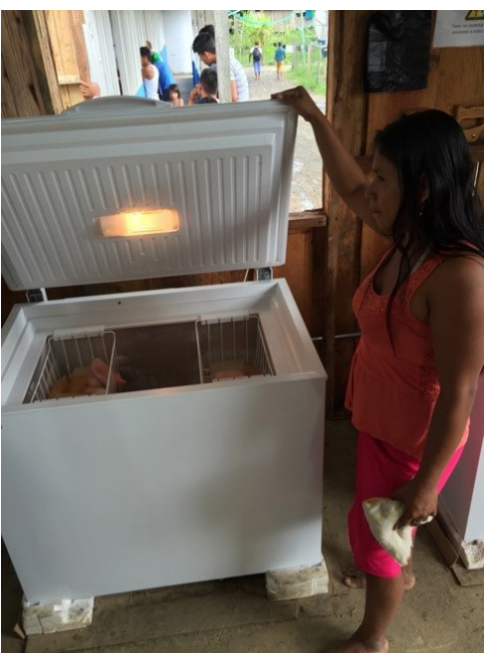

b.

Figura 1. a. Diagrama unifilar de la escuela. b. Unidad componente del sistema de refrigeración

Fuente: elaboración propia.

Tabla 2. Descripción del sistema actual

\begin{tabular}{cccc}
\hline Símbolo & Parámetro & Valor & Unidad \\
\hline & Potencia máxima & 213,15 & $\mathrm{~W}$ \\
\hline $\mathrm{V}_{\mathrm{OC}}$ & Voltaje circuito abierto & 36,3 & $\mathrm{~V}$ \\
\hline $\mathrm{I}_{\mathrm{CC}}$ & Corriente de corto circuito & 7,84 & $\mathrm{~V}$ \\
\hline $\mathrm{V}_{\mathrm{mp}}$ & Tensión en el máximo punto de potencia & 29 & $\mathrm{~A}$ \\
\hline $\mathrm{I}_{\mathrm{mp}}$ & Corriente en el punto máximo de potencia & 7,35 & ${ }^{\circ} \mathrm{C}$ \\
\hline $\mathrm{T}$ & Temperatura de la celda & 25 & \\
\hline
\end{tabular}

Fuente: elaboración propia. 


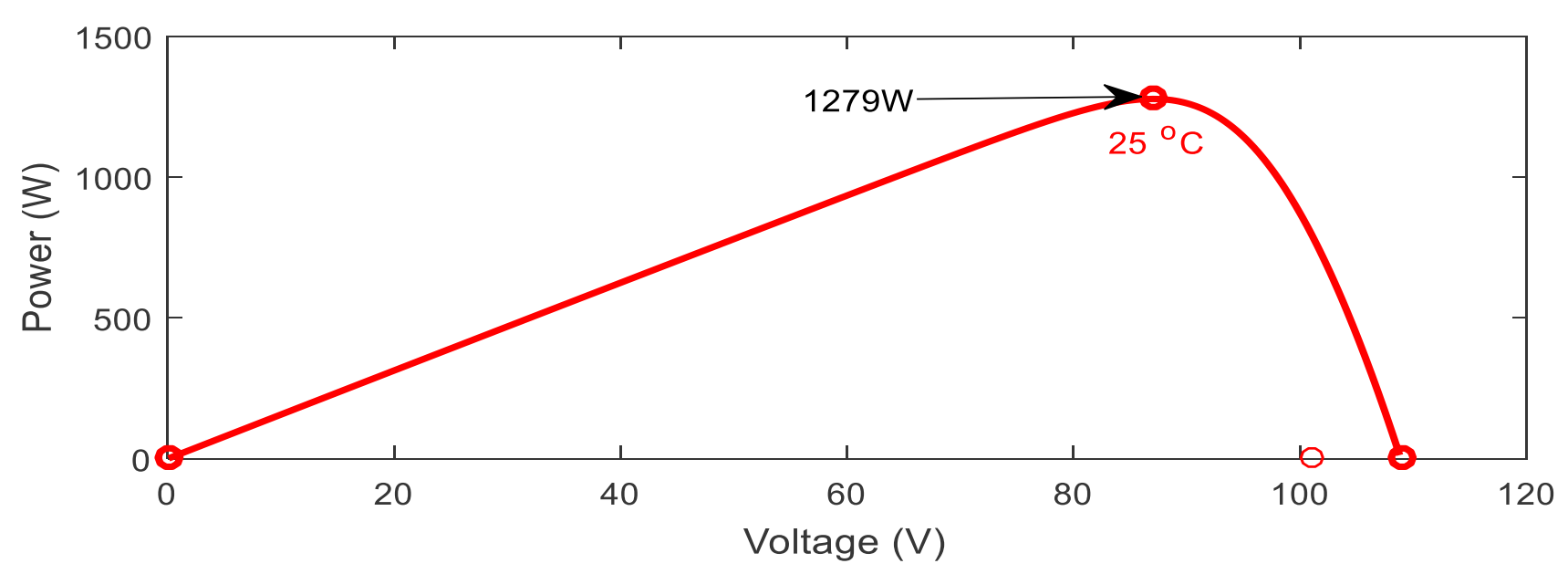

Figura 2. Simulación arreglo PV escuela. Configuración de paneles; 3 en serie y 2 paralelos Fuente: elaboración propia.

La figura 4. demuestra que la demanda del centro de refrigeración es cubierta por los paneles fotovoltaicos en los periodos donde la irradiación es mayor o igual a $1000 \mathrm{~W} / \mathrm{m}^{\wedge} 2$. Los parámetros del arreglo FV establecidos para obtener una potencia máxima de 837,9 W son los de la tabla 2, ya que se utiliza el mismo panel del sistema de la escuela.

\section{ANÁLISIS DE ESTABILIDAD}

El análisis de estabilidad fue desarrollado mediante el simulador NEPLAN. Una vez mode- lado el sistema a partir de los valores nominales de los equipos y la carga, se realiza el flujo de carga para identificar los valores de operación y el correcto funcionamiento del sistema.

Las figuras 5 y 6 . demuestran que los sistemas operan correctamente en estado estacionario, porque es claro que el flujo converge, los valores de tensión se mantienen en el rango esperado y no se presenta sobrecarga en las líneas y equipos. a.

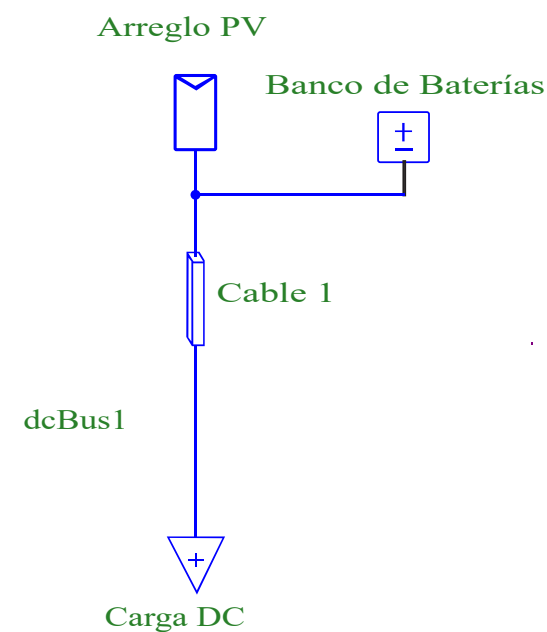

b.

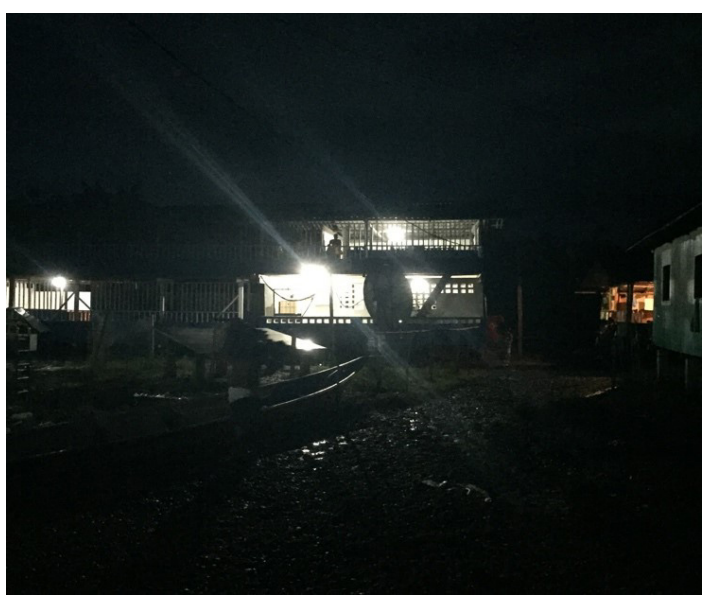

Figura 3. a. Diagrama unifilar del centro de refrigeración. b. Visión nocturna del centro educativo con servicio de energía eléctrica

Fuente: elaboración propia. 


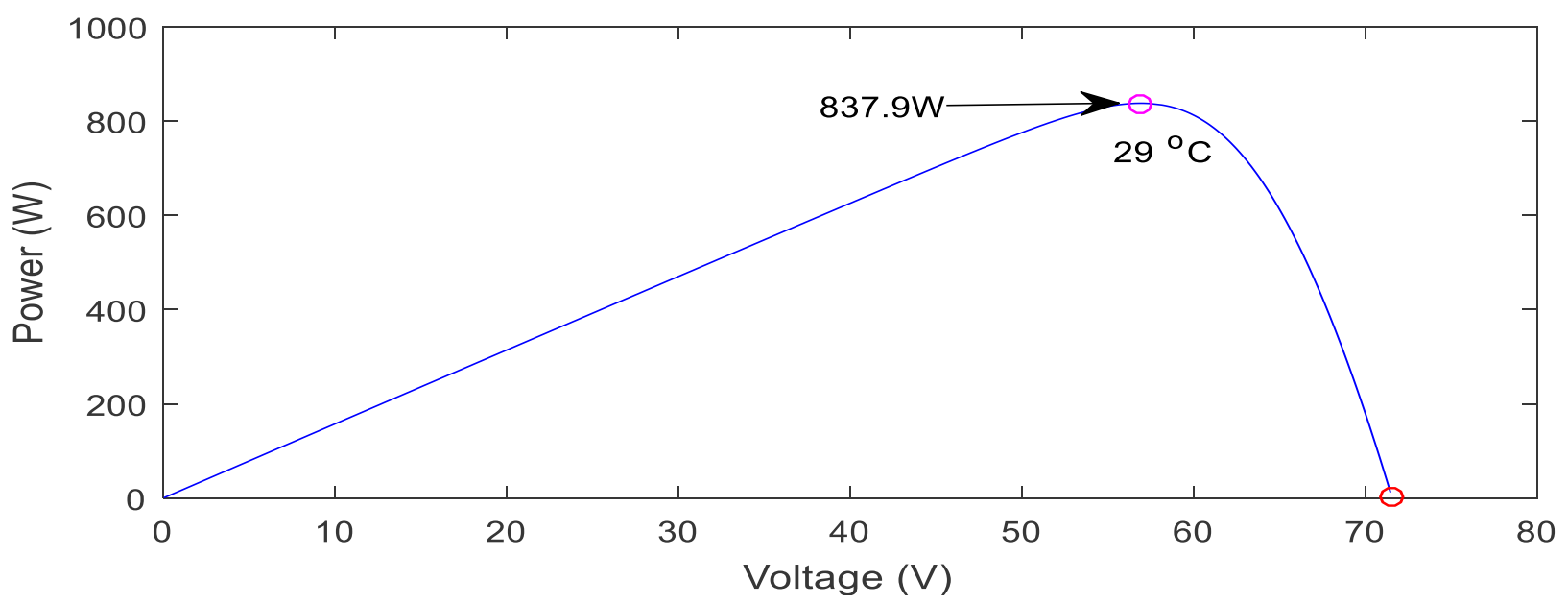

Figura 4. Simulación arreglo PV centro de refrigeración. Configuración de paneles; 2 en serie y 2 paralelos.

Fuente: elaboración propia.

Para verificar la estabilidad del sistema se analiza la estabilidad de pequeña señal en Neplan. El resultado se ilustra en la figura 7 , donde se demuestra que tanto el sistema de la escuela (7a) como el del centro de refrigeración (7b) son estables ante los parámetros de generación y carga establecidos para cada uno. Es decir, la carga se alimenta adecuada- mente y el suministro de energía es constante (Dias, Garcés y González, 2017).

\section{PROPUESTA DE EXPANSIÓN}

A partir de la microrred instalada en el resguardo indígena se realiza una propuesta de expansión para aumentar la cobertura

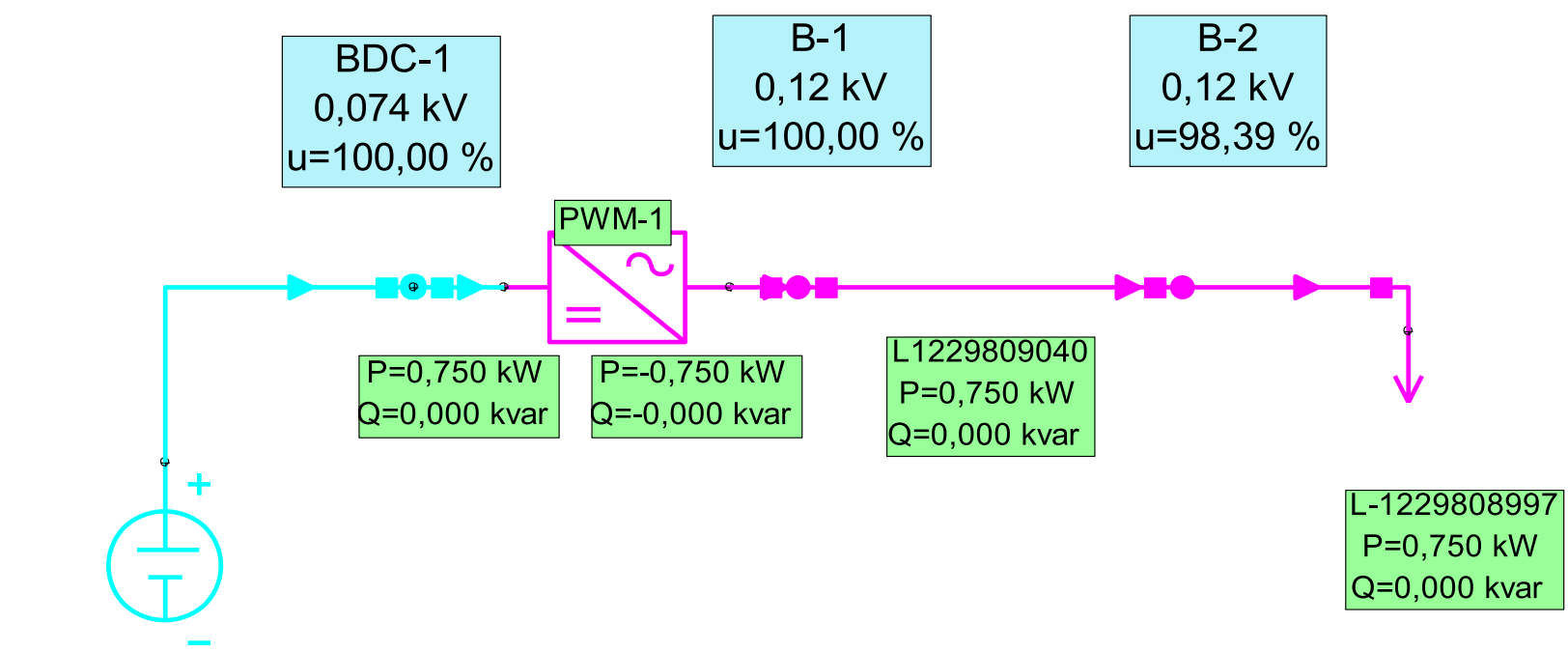

DCBAT-1229809171

$P=-0,750 \mathrm{~kW}$

$\mathrm{Q}=0,000 \mathrm{kvar}$

Figura 5. Flujo de carga del sistema instalado en la escuela.

Fuente: elaboración propia. 


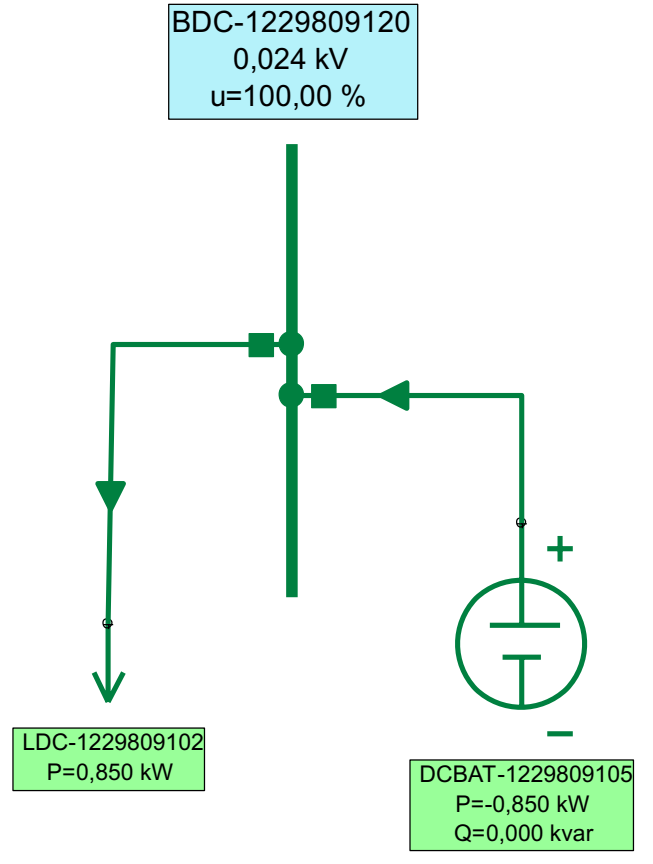

Figura 6. Flujo de carga del sistema en el centro de refrigeración

Fuente: elaboración propia.

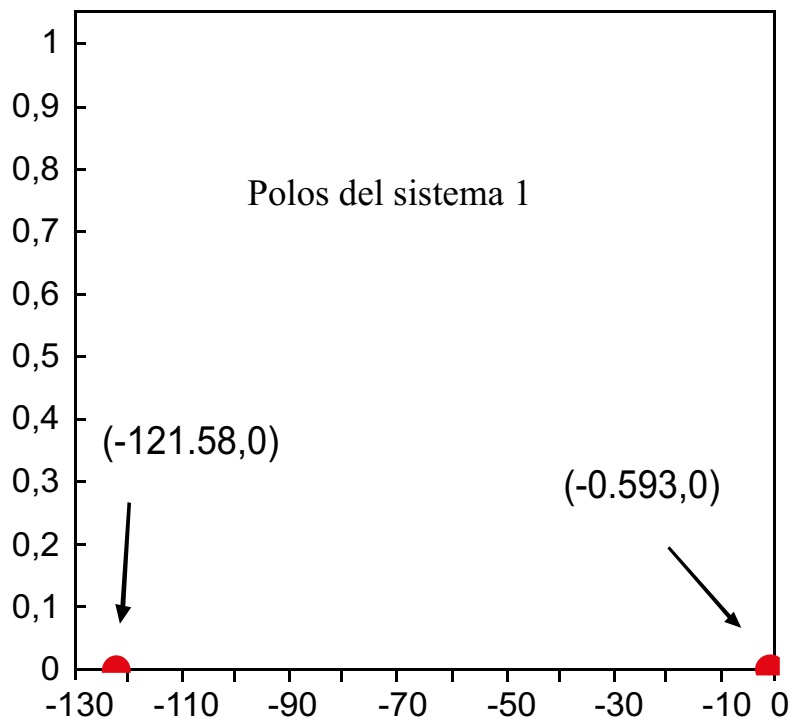

a) del sistema. Se llevan a cabo dos casos de expansión para determinar cuál de los dos sería más viable. En consecuencia, se tiene en cuenta el acople de los dos sistemas de generación a un mismo nodo y el aumento de generación de los paneles fotovoltaicos para dejar un solo centro de generación. Es importante resaltar que la expansión de la microrred busca abastecer a más usuarios y aumentar la confiabilidad del sistema.

\section{Caso I}

En el primer caso se presenta una propuesta de acoplamiento entre los dos sistemas existentes, como se muestra en la figura 8.

El sistema acoplado se modela teniendo en cuenta el nodo DC donde se conectarán cargas netamente de corriente continua y el inversor DC/AC el cual hará la conversión a corriente alterna para conectar las cargas AC que sean requeridas.

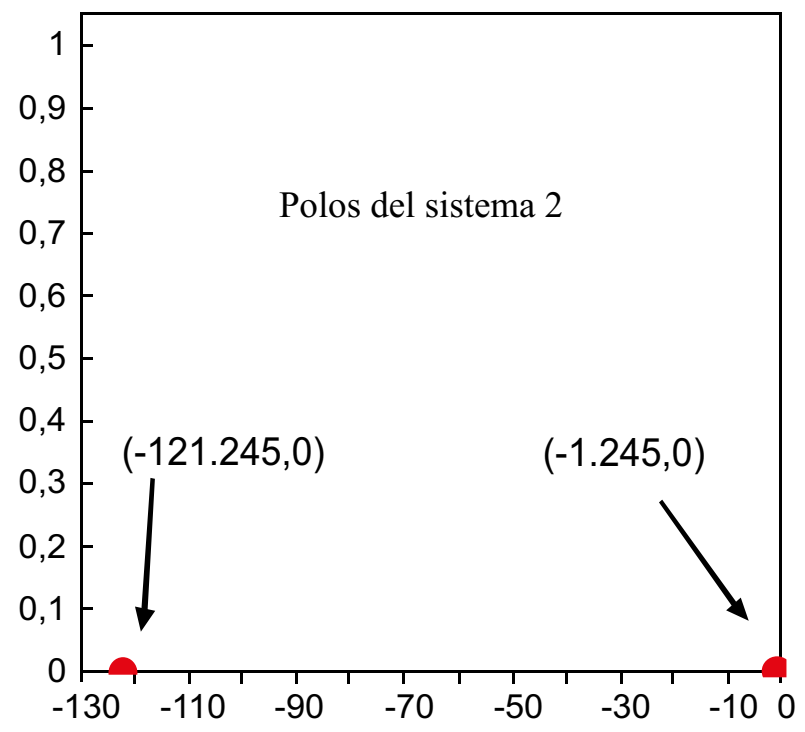

b)

Figura 7. a. Estabilidad del sistema de la escuela. b. Estabilidad del centro de refrigeración Fuente: elaboración propia. 
Al realizar el flujo de carga se puede observar que este cumple adecuadamente y es coherente; es decir, converge, y las tensiones en las barras es correcta, puesto que sus valores están en 0,98 y 1 en p.u. Además, no se presentan elementos ni líneas sobrecargadas, lo cual indica que su implementación en el software es exitosa y logra suplir la de- manda tanto de la carga DC como AC, a la vez que mantiene las condiciones técnicas de la microrred.

Es importante resaltar que al realizar el acople se busca aumentar la potencia de generación y con base en dicha potencia es que se lleva a cabo la implementación en el software y se

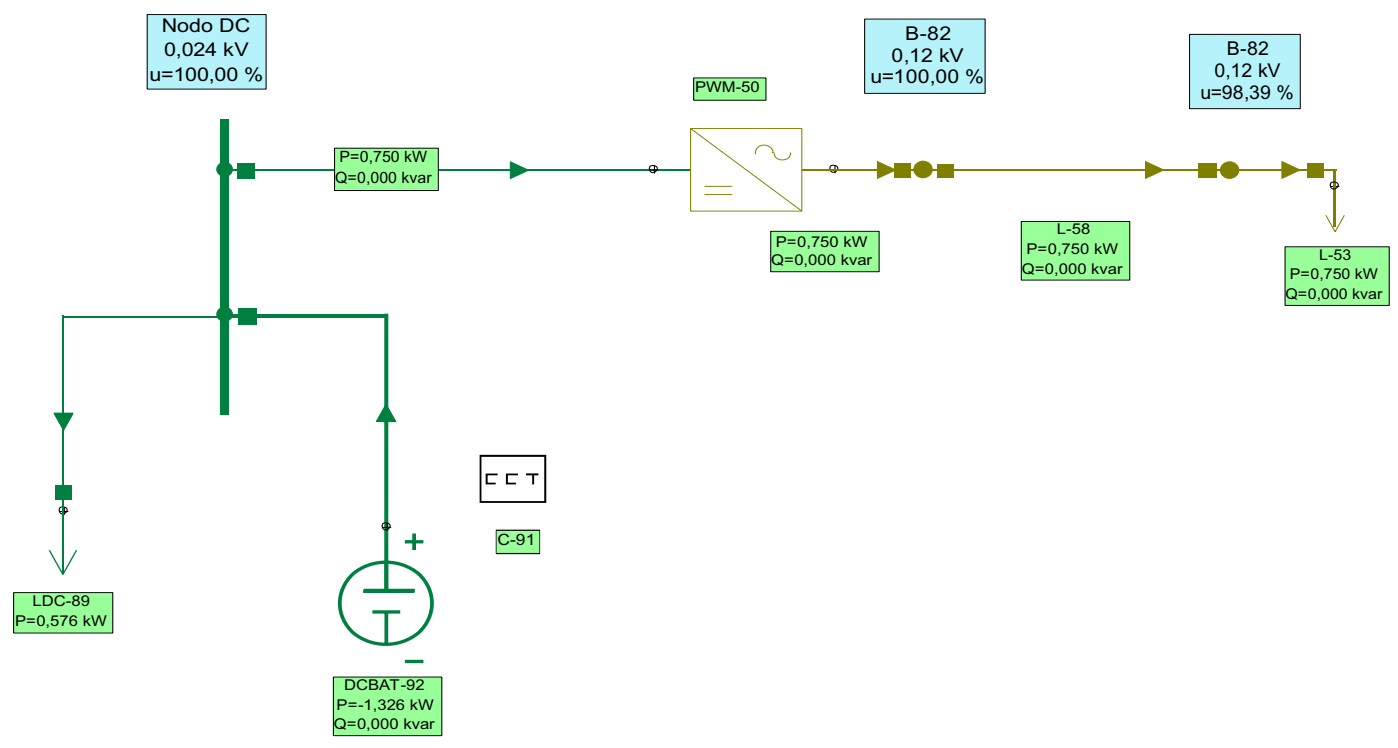

Figura 8. Acople de los dos sistemas.

Fuente: elaboración propia.

simula con una única fuente de generación la cual es dada por el acople de los arreglos fotovoltaicos. Por otro lado, cabe resaltar que los bancos de baterías quedan independientes precisamente para asegurar un respaldo en caso de que alguno de los dos falle.

Posteriormente se analiza la estabilidad de pequeña señal por medio de la herramienta que se encuentra en Neplan, como se hizo para los sistemas 1 (sistema escuela) y 2 (sistema centro de refrigeración). De esto se obtuvo una ubicación de polos como se muestra en la figura 9.

De la figura 9. se puede determinar que la propuesta de expansión cuenta con dos polos en el semiplano complejo izquierdo en las coordenadas $(-121,9 ; 0)$ y $(-0,978 ; 0)$. Esto indica que el sistema es estable, es decir, que las cargas se están alimentando adecuadamente y el suministro de energía es constante. Además, el sistema, a pesar de que cuenta con cargas DC y AC, funciona correctamente.

Al considerar un aumento de carga, se busca demostrar que el sistema es robusto ante pequeñas perturbaciones, es decir, mantiene su estabilidad sin importar dichos cambios. Esto se refleja en el análisis de pequeña señal que se realiza al aumentar cargas DC y AC en el sistema, como se muestra en la figura 10.

Al aumentar la carga se observa en la figura 11. que el sistema se mantiene estable, 


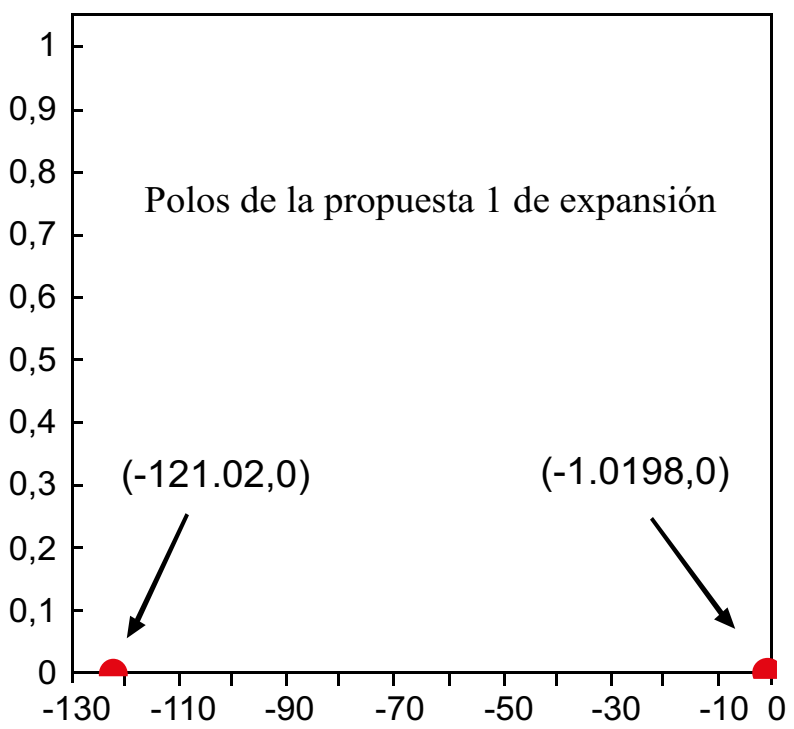

Figura 9. Valores propios al unir los dos sistemas

Fuente: elaboración propia.

esto se determina a partir de la ubicación de los polos. En este caso existe un polo más pero no aparece en la figura debido a que su valor es muy pequeño $(-0,018$; $0)$. Por otro lado, los otros polos están en las coordenadas $(-0,508 ; 0)$ y $(-121,244 ; 0)$ respectivamente. Los valores son similares a los obtenidos en la figura 9. Pero estos varían con el aumento de carga y su diferencia radica en que la perturbación del sistema es muy pequeña. Esto cumple con el concepto del análisis de estabilidad de pequeña señal. Luego de comprobar que el sistema al aumentar las cargas lineales mantiene su estabilidad, se realiza la prueba conectando una carga no lineal al nodo AC, esta carga tiene las características mostradas en la tabla 3.

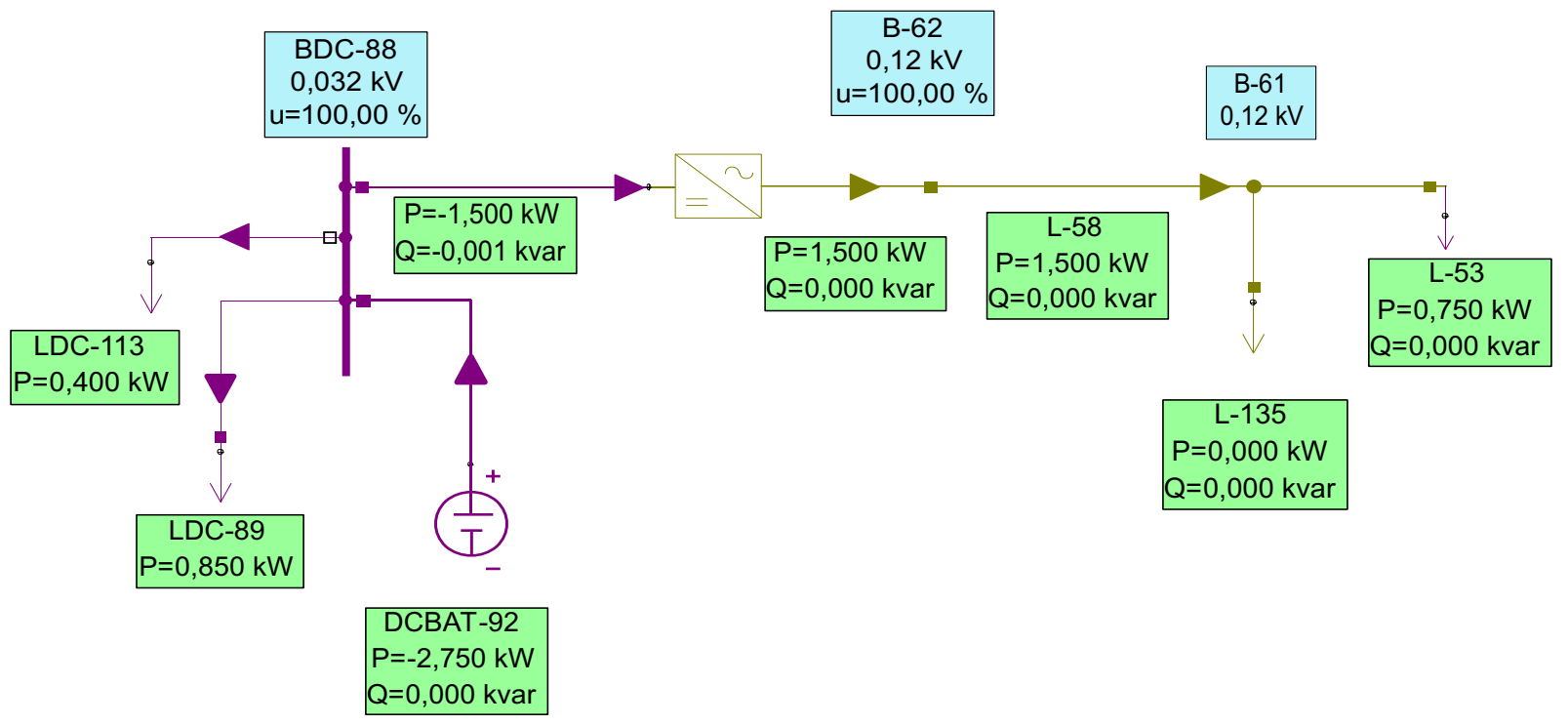

Figura 10. Efecto del aumento de carga Fuente: elaboración propia. 
La potencia reactiva positiva indica que la carga es de tipo inductivo. Al realizar el flujo de potencia con la nueva carga se aumen- ta la potencia de la fuente, debido a que la conexión de esta nueva carga demanda más energía; es importante resaltar que no se ve

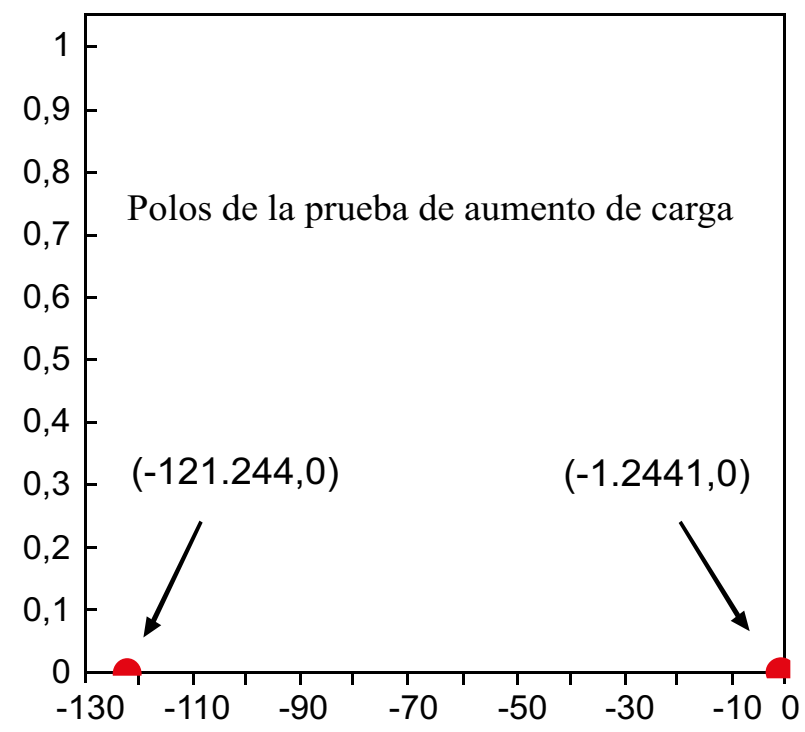

Figura 11. Valores propios del aumento de carga Fuente: elaboración propia.

afectada la convergencia del flujo, y este cumple correctamente como en los casos anteriormente mencionados. Luego se lleva a cabo el análisis de pequeña señal, del cual se obtienen los valores propios del sistema, como se muestra en la figura 12. la posición de los polos in-

Tabla 3. Características de carga no lineal AC

\begin{tabular}{cc}
\hline Tipo de nodo & PQ \\
\hline $\mathrm{S}(\mathrm{kVA})$ & 0,071 \\
\hline $\mathrm{P}(\mathrm{kW})$ & 0,05 \\
\hline $\mathrm{Q}(\mathrm{kvar})$ & 0,05 \\
\hline $\mathrm{I}(\mathrm{A})$ & 0,34 \\
\hline Cos (phi) & 0,707 \\
\hline
\end{tabular}

Fuente: elaboración propia. dica que el sistema no pierde la propiedad de estabilidad ante dicha perturbación.

\section{Caso II}

Para el segundo caso se propone un solo centro de generación. Este sistema dispone de 12 paneles fotovoltaicos YL250P-29b de 250 $W p$, con una configuración de seis conjuntos de paneles en paralelo, donde cada uno consta de dos paneles en serie. La figura 13. demuestra que la demanda del resguardo indígena es cubierta por los paneles solares en los periodos donde la irradiación es mayor o igual a $1000 \mathrm{~W} / \mathrm{m}^{\wedge} 2$, debido a que se puede llegar a generar una potencia de $3006 \mathrm{~W}$ frente a una potencia demanda de $2800 \mathrm{~W}$.

El análisis de este sistema fotovoltaico se lleva a cabo mediante el simulador Neplan. Se modela con una fuente DC, un inversor DC/AC, una carga AC (1950 W) y una carga DC $(850 \mathrm{~W})$. En el desarrollo de este caso se 


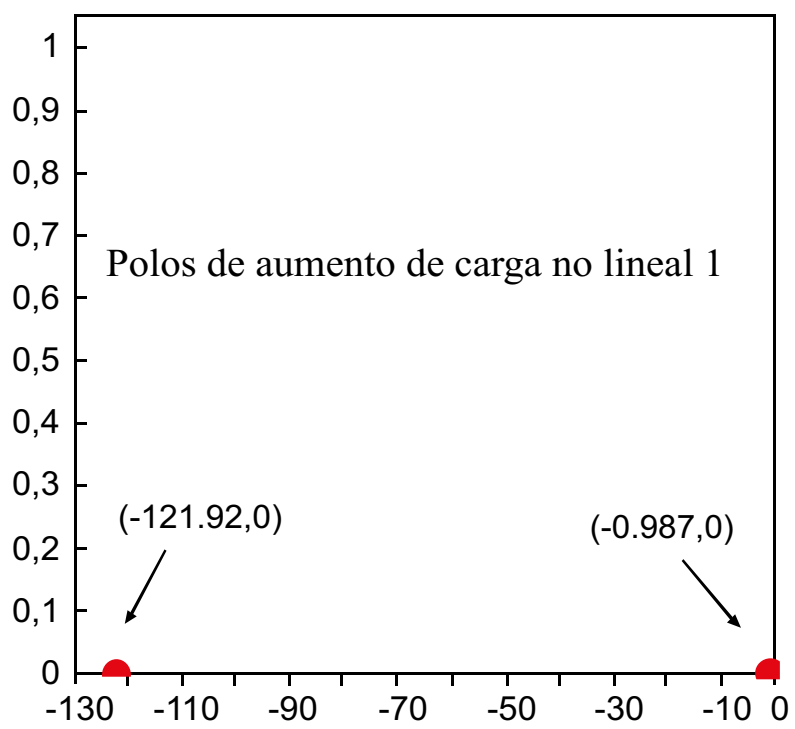

Figura 12. Valores propios del aumento carga no lineal caso 1 Fuente: elaboración propia.

plantea un banco de baterías como la fuente de generación, por tanto, la potencia activa suministrada no es la generada por los paneles FV sino la demanda por la carga, es decir $2800 \mathrm{~W}$.

Las baterías que se proponen para este caso son las MT122550 MTEK. Estas operan a 12 $\mathrm{V}$ y tienen una capacidad de 255 Ah C10. Es decir, pueden suministrar 25,5 A constantes durante 10 horas de funcionamiento. Por esto, se propone instalar 10 baterías conectadas en cinco conjuntos en paralelo y en cada uno dos baterías conectadas en serie; así pues, se proporciona $24 \mathrm{~V}$ al sistema y una capacidad de 127,5 A. El centro de refrigeración funciona directamente desde el nodo de $24 \mathrm{~V}$, esta carga opera en DC, por tanto, no es necesario instalar un inversor en esta parte del sistema.

Dado que muchas de las cargas en el resguardo indígena requieren alimentación $\mathrm{AC}$, para alumbrado y electrodomésticos de primera necesidad, se debe utilizar un inversor en el sistema. El voltaje requerido

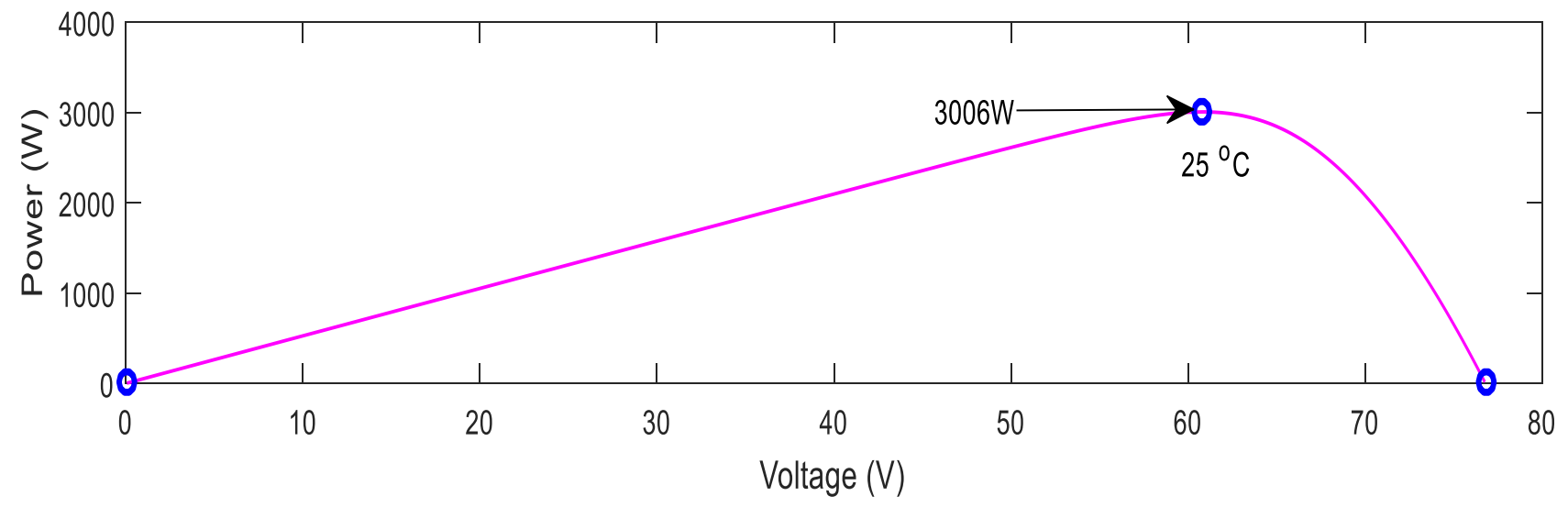

Figura 13. Simulación arreglo PV centro de generación. Configuración de paneles; 3 en serie y 5 paralelos Fuente: elaboración propia. 
es de 120 Vac, por consiguiente, se debe aumentar el voltaje además del cambio de corriente continua a corriente alterna. Se propone un inversor con tensión de entrada de $24 \mathrm{Vdc}$ y potencia de salida 2000 W. Una vez implementado el sistema en Neplan, se ejecuta el flujo de carga para analizar el sistema eléctrico en estado estacionario. La figura 14. demuestra que el planteamiento de expansión del sistema opera correctamente, y se obtiene la magnitud del voltaje en cada nodo, y la potencia activa y reactiva que fluye en cada línea.
Para el análisis de estabilidad de pequeña señal se tuvo en cuenta la cargabilidad máxima del sistema. Es decir, la mayor demanda de potencia que se puede presentar en la microrred. En la figura 15, se muestra el resultado obtenido en Neplan del análisis de estabilidad de pequeña señal, donde se observa los valores propios con su componente real negativa, correspondientes al sistema propuesto para este caso. Se considera un sistema estable, debido a que los polos están ubicados en el semiplano imaginario negativo.

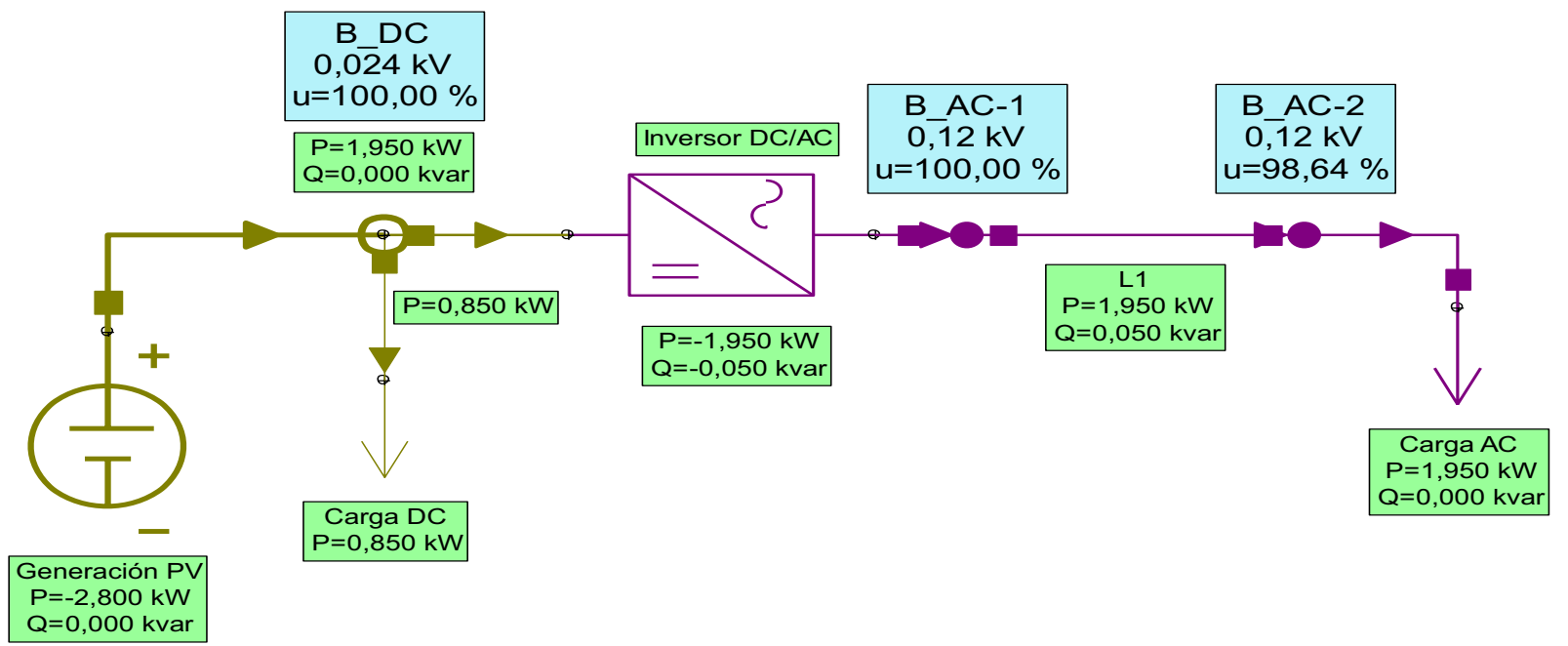

Figura 14. Arquitectura y simulación de flujo de carga del caso 2 en Neplan Fuente: elaboración propia.

Luego de comprobar que el sistema con cargas lineales es estable, se adelanta una prueba con una carga no lineal, la cual presenta las características mostradas en la tabla 4. Se reconoce que la nueva carga conectada es inductiva porque presenta un factor de potencia de 0,81 y la potencia reactiva absorbida es de magnitud positiva. La figura 16. demuestra que al conectar una carga in- ductiva el sistema opera correctamente, es decir, la tensión en cada uno de los nodos se mantiene en los valores ideales de operación y la generación es suficiente para abastecer la nueva demanda. Al analizar la estabilidad de pequeña señal se determina que el sistema es estable al conectar este tipo de carga, ya que sus polos están ubicados en el semiplano imaginario ne- 
gativo, como se evidencia en la figura 17. También se puede observar que el polo dominante $(-0,004 ; 0)$ se ubica una posición más a la derecha después de haber conectado la nueva carga; el cambio no es tan significativo. Por esta razón, la propiedad de estabilidad en el sistema se mantiene frente a pequeñas perturbaciones.

\section{CONCLUSIONES}

El análisis de estabilidad de pequeña señal es ideal para estudiar el comportamiento de una microrred aislada ante pequeñas perturbaciones en la generación o carga. Es decir, las propuestas de modificación

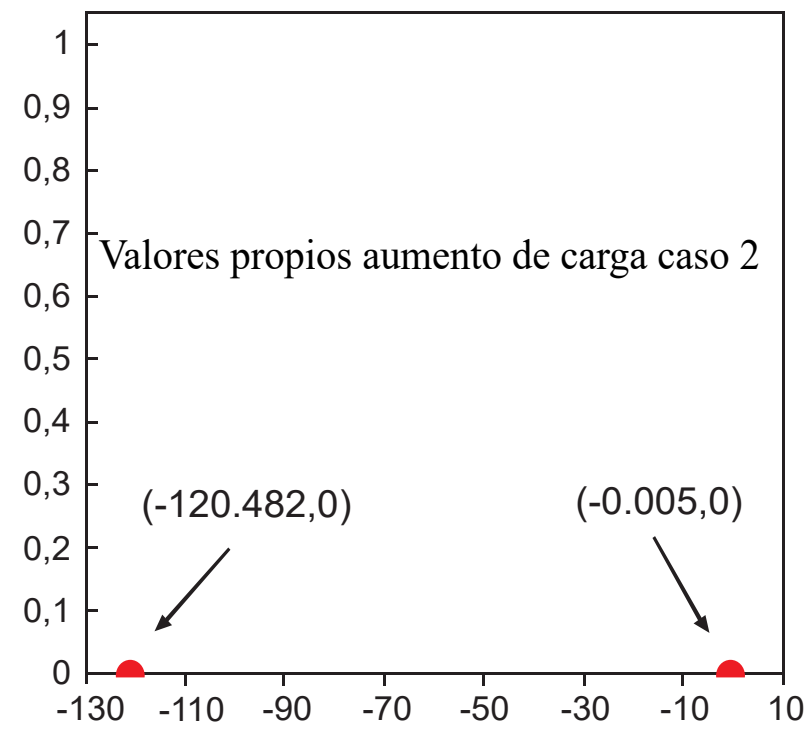

Figura 15. Valores propios caso 2

Fuente: elaboración propia.

Tabla 4. Características de carga no lineal AC

\begin{tabular}{cc}
\hline Tipo de nodo & PQ \\
\hline $\mathrm{S}(\mathrm{kVA})$ & 0,086 \\
\hline $\mathrm{P}(\mathrm{kW})$ & 0,07 \\
\hline $\mathrm{Q}(\mathrm{kvar})$ & 0,05 \\
\hline $\mathrm{I}(\mathrm{A})$ & 0,41 \\
\hline Cos (phi) & 0,814 \\
\hline
\end{tabular}

Fuente: elaboración propia. 


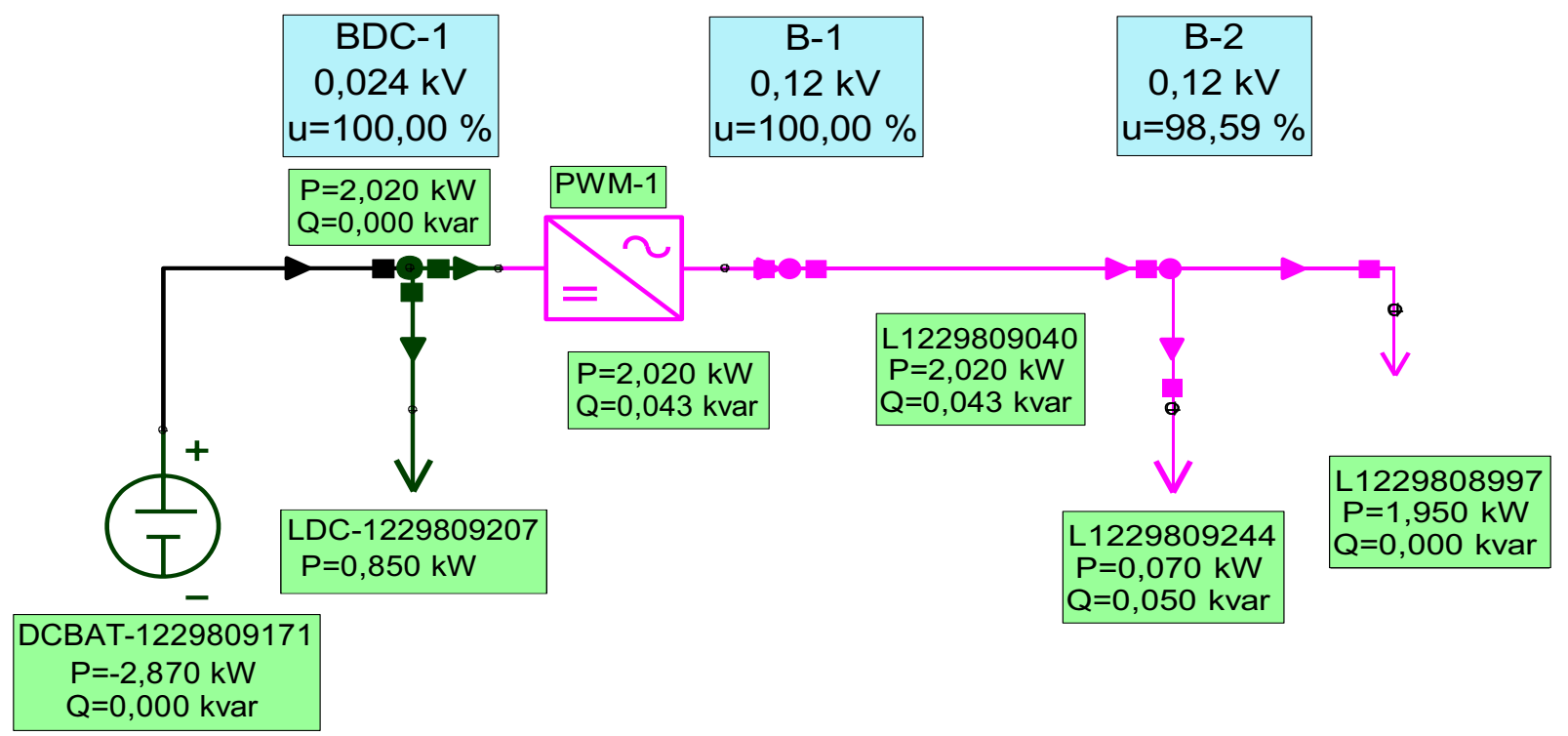

Figura 16. Flujo de carga del caso 2 con carga inductiva Fuente: elaboración propia.

o expansión de microrredes deben ser estudiadas por medio del análisis de estabilidad de pequeña señal, debido a que estas se clasifican como pequeñas perturbaciones, y a que los valores nominales deoperación son poco significativos pequeños en comparación a un sistema de potencia convencional. De las dos alternativas de expansión propuestas, se determina que el caso 1 es el más viable, ya que al tener una microrred con dos fuentes de generación ofrece mayor confiabilidad; así, cada una

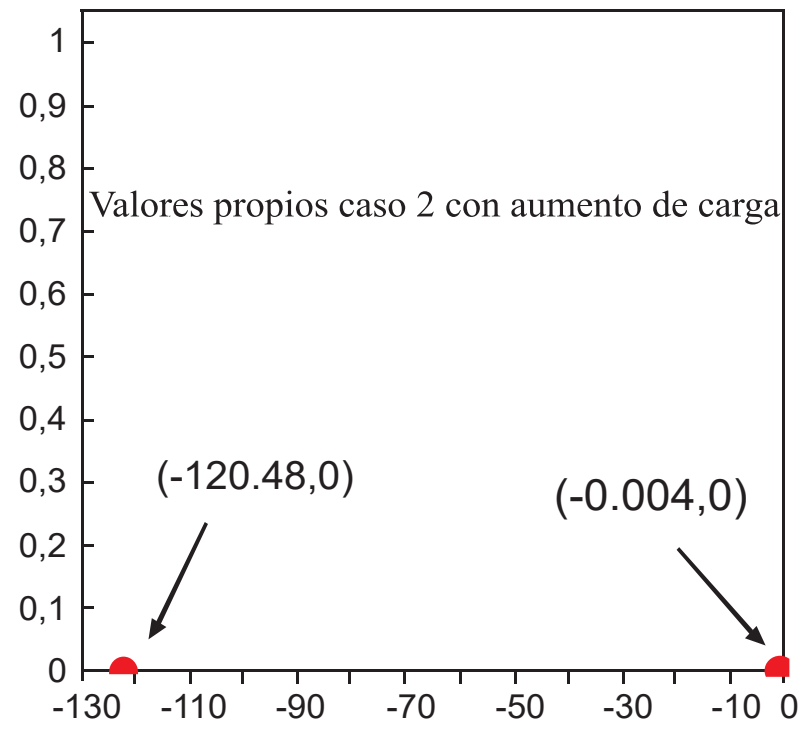

Figura 17. Valores propios del caso 2 con carga inductiva Fuente: elaboración propia. 
de las unidades de generación se pueden conectar al sistema de forma individual o conjunta, según la cargabilidad, o incluso funciona de respaldo en caso de que uno de las dos falle.

Los casos 1 y 2 demuestran que pueden operar de manera equilibrada, es decir, conservan el balance entre carga y generación frente a condiciones de cargabilidad máxima; incluso, después de haber sido sometidas a alguna perturbación (variaciones en la carga o en la generación) estas no pierden la propiedad de estabilidad.

\section{REFERENCIAS}

Aabrek, V., Forseth, I. Bueno-López, M. y Molinas, M. (2018). Design and Implementation of a Monitoring System for Decision Support in a Micro-Business Based on Solar Energy Microgrid in Rural Colombia. En IEEE Global Humanitarian Technology Conference (GHTC) (pp. 1-8). San José, CA. DOI: 10.1109/ GHTC.2018.8601571

Bastidas, J., Oviedo, J. y Solano, J. (2017). Técnicas de análisis y control para la mejora de la estabilidad de microrredes eléctricas: revisión en la literatura. En IX Simposio Internacional Sobre Calidad de La Energía Eléctrica (SICEL) (pp. 1-10). Bucaramnga, Colombia.

Bueno-López, M. y Garzón-Lemos, S. (2017). Electrification in Non-Interconnected Areas: Towards a New Vision of Rurality in Colombia. IEEE Technology and Society Magazine, 36(4), 73-79. DOI: 10.1109/MTS.2017.2763479

Correa F., C.A., Marulanda G., G.A. y Panesso H., A.F. (2017). Impacto de la pene- tración de la energía solar fotovoltaica en sistemas de distribución: estudio bajo supuestos del contexto colombiano. Tecnura, 20(50), 85-95. DOI: https://doi. org/10.14483/22487638.11563

Dias T., D.A., Garcés R., A. y González O., D. (2017). Despacho económico en sistemas de potencia considerando estabilidad transitoria. Tecnura, 21(51), 27-41. DOI: https://doi.org/10.14483/udistrital. jour.tecnura.2017.1.a02

Giral R., W.M., Celedón F., H.J., Galvis R., E. y Zona O., A.T. (2017). Redes inteligentes en el sistema eléctrico colombiano: Revisión de tema. Tecnura, 21(53), 119-137. DOI: https:// doi.org/10.14483/22487638.12396

He, J., Wu, X., Xu, Y. y Guerrero, J.M. (2019). Small-Signal Stability Analysis and Optimal Parameters Design of Microgrid Clusters. IEEE Access, (7), 36896-36909. DOI: 10.1109/ACCESS.2019.2900728

Louie, H. (2018). Off-Grid Electrical Systems in Developing Countries. Seattle, WA: Springer International Publishing.

Majumder, R. (2013). Some aspects of stability in microgrids. IEEE Transactions on Power Systems, 28(3), 3243-3252. DOI: https:// doi.org/10.1109/TPWRS.2012.2234146

Ramírez, M. y Aquino, R.A. (2009). Análisis de estabilidad de pequeña señal en el sistema eléctrico de Pereira. [Tesis de pregrado]. Universidad Tecnológica de Pereira, Pereira. Recuperado de http:// repositorio.utp.edu.co/dspace/bitstream/ handle/11059/1986/6213743D946.pdf 
Song, Y., Hill, D.J. y Liu, T. (2019). Impact of DG Connection Topology on the Stability of Inverter-Based Microgrids. IEEE Transactions on Power Systems, 34(5), 3970-3972. DOI: 10.1109/ TPWRS.2019.2917624

Stevanoni, C., De Grève, Z., Vallée F. y Deblecker, O. (2019). Long-Term Planning of Connected Industrial Microgrids: A Game Theoretical Approach Including Daily Peer-to-Microgrid Exchanges. IEEE Transactions on Smart Grid, 10(2), 22452256. DOI: 10.1109/TSG.2018.279331

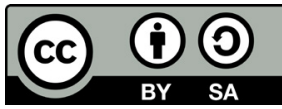

